\title{
Crystal landscape of chiral drug Chlorphenesin and its structural analogues: polymorphism of racemic and enantiopure samples, metastable and stable racemic conglomerates, diverse in unity crystal motifs
}

\section{Supporting information}

Alexander A. Bredikhin*, Dmitry V. Zakharychev, Aidar T. Gubaidullin, Aida I. Samigullina, and Zemfira A. Bredikhina

Arbuzov Institute of Organic and Physical Chemistry, FRC Kazan Scientific Center of RAS, Arbuzov St., 8, Kazan 420088, Russian Federation

Corresponding Author: $\quad$ Alexander A. Bredikhin (baa@iopc.ru)

Graphical presentation of the free energy ratios and eutectic ratios of stable and metastable phases for chlorphenesin 1 and its meta- and ortho-substituted analogs 2 and 3, calculated on the basis of DSC data

The objects of this study were the chiral drug Chlorphenesin 1 and its meta- and orthosubstituted analogs $\mathbf{2}$ and $\mathbf{3}$.<smiles>OCC(O)COc1ccc(Cl)cc1</smiles>

1<smiles>OCC(O)COc1cccc(Cl)c1</smiles>

2

3

Based on the obtained thermochemical parameters of the identified crystalline forms for compounds 1-3, as well as DSC measurements of the dependence of the heat capacity of these forms on temperature in the range $20 \div 100{ }^{\circ} \mathrm{C}$, the curves of the change in the free energies of these forms were calculated. The calculation methodology and the assumptions made in this case are described in our previous works. ${ }^{1-3}$ The results obtained are presented in Figures S1-S3. 


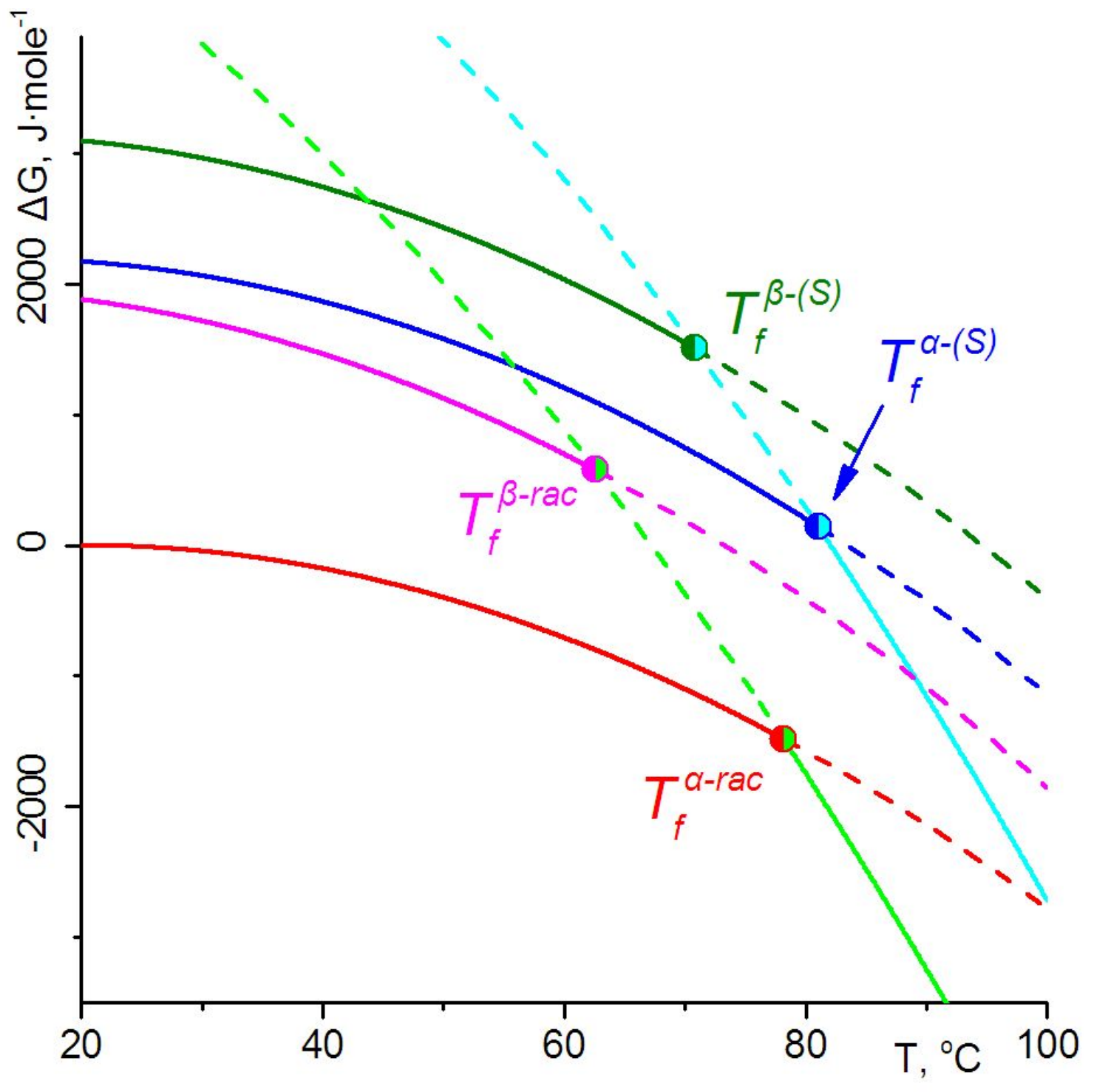

Figure S1. Dependence of the change in the free energies of the phases identified in system 1 on temperature: red curve - $\alpha-r a c-1$, magenta curve - $\beta$-rac-1, blue curve - $\alpha-(S)$-1, olive curve - $\beta-(S)$ 1 , green curve and cyan curve - racemic and enantiopure melts, respectively. In the region indicated by the dotted line, each of the phases is thermodynamically unstable; phases $\beta-r a c-1$ and $\beta-(S)-\mathbf{1}$ are metastable throughout the interval where they are observed. 


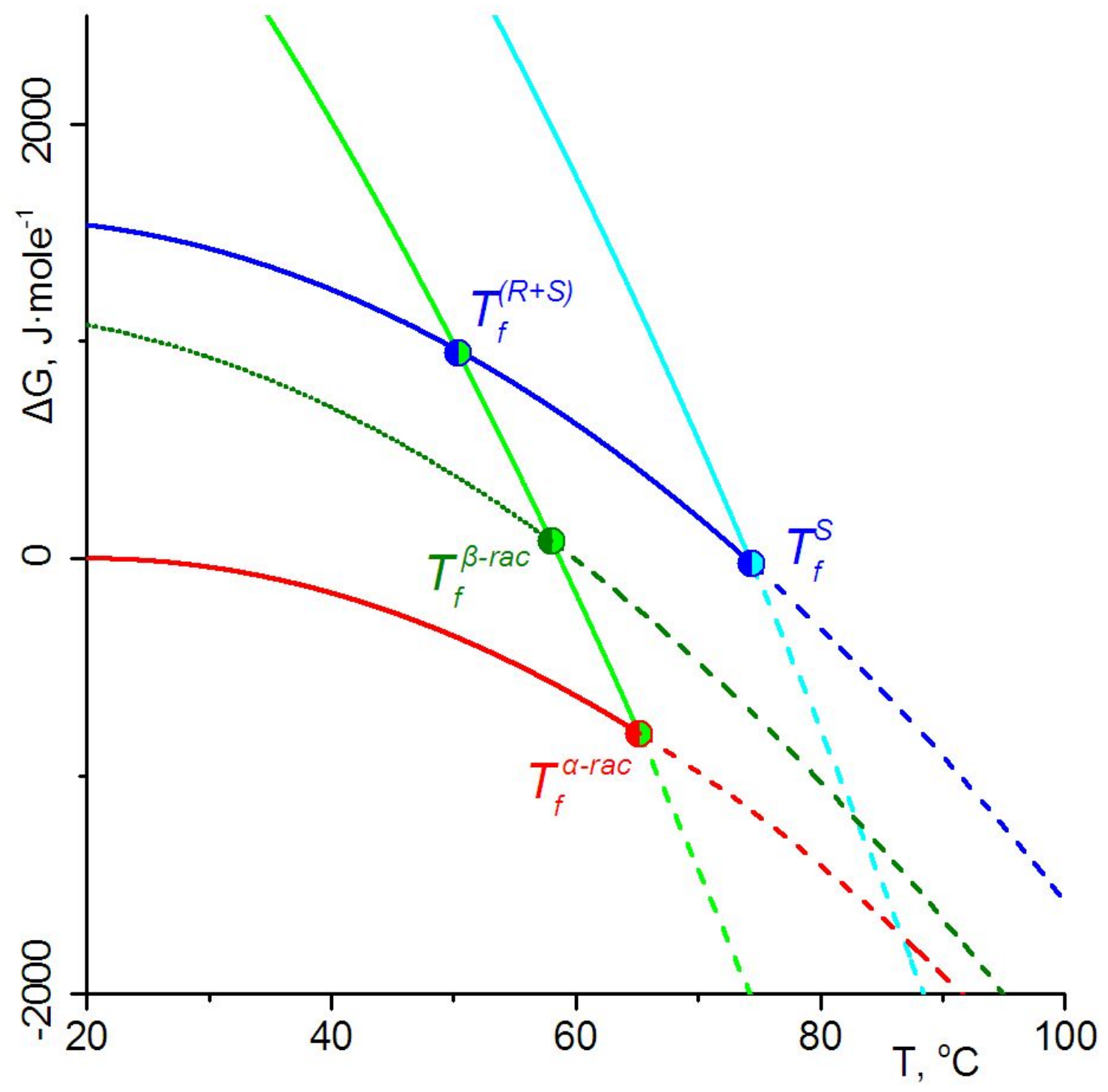

Figure S2. Dependence of the ratio of free energies of phase states of compound 2 on temperature: $\alpha$-rac-2 (red curve), $\beta$-rac-2 (olive curve), enantiopure $S$-isomer (blue curve), racemic melt (light green curve), scalemic melt (light blue curve). In the region indicated by the dotted line, each of the phases is thermodynamically unstable; the $\beta-r a c-2$ phase is metastable throughout the interval where it is observed. 


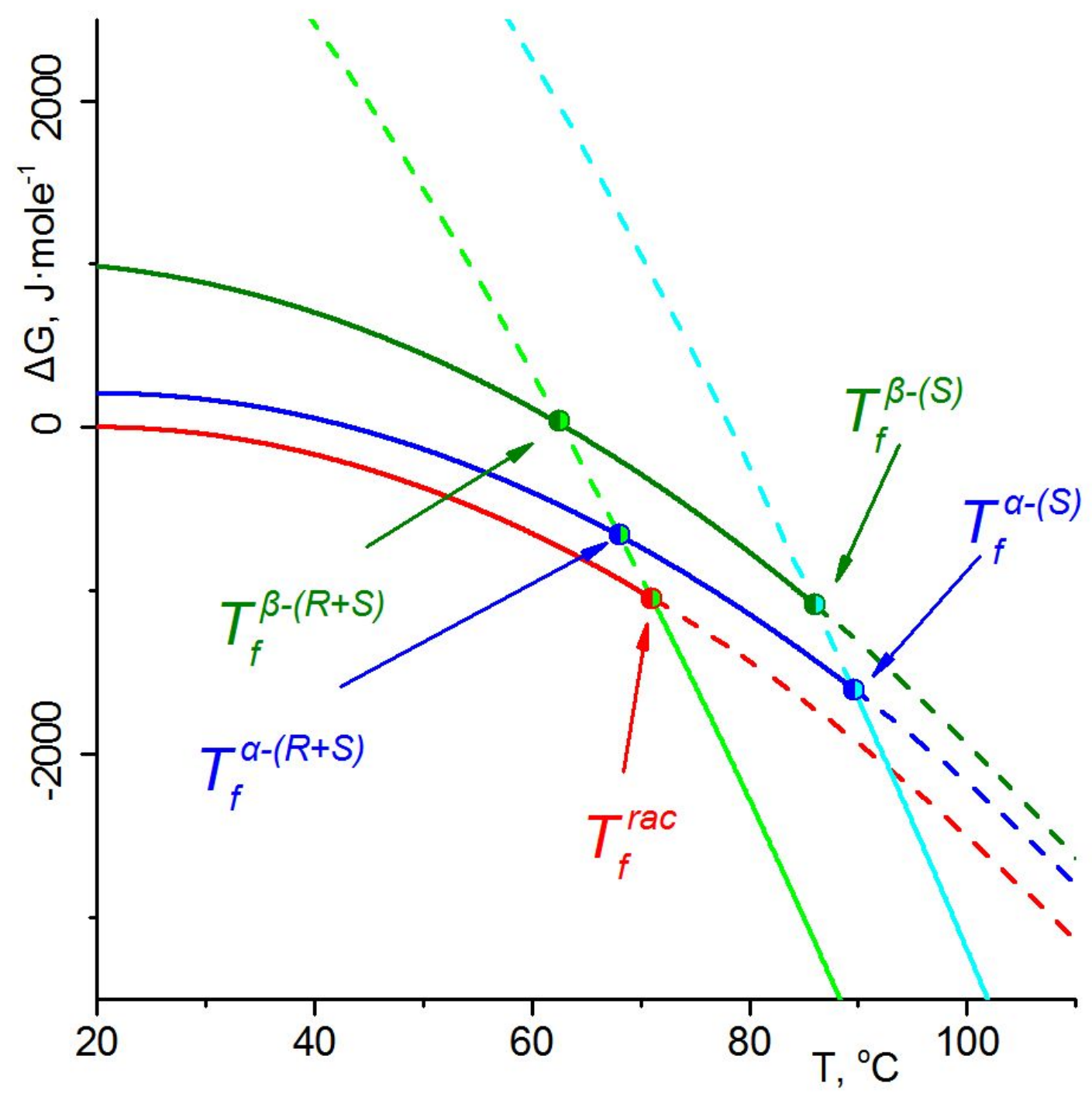

Figure S3. Dependence of the free energy ratios of diol 3 phase states on temperature: $\alpha-r a c-3$ (red curve), $\alpha-(S)-3$ (blue curve), $\beta-(S)-3$ (olive curve), racemic melt (green curve), scalemic melt (light blue curve). In the region indicated by the dotted line, each phase is thermodynamically unstable. The $\beta-(S)-3$ phase is metastable throughout the interval where it is observed.

Energy diagrams displaying the temperature dependences of the ratios between the free energies of the phases constituting the system make it possible to visualize the stability regions of these phases, as well as phase transitions realized in such a system, incl. under conditions of metastability. But such diagrams in their traditional form do not reflect the thermochemical behavior of the system to the full. In particular, they show the melting points of individual (incl. metastable) phases, but there is no representation of eutectic melting points. Phase diagrams are usually used to represent the latter, but their information content is much lower, and they do not 
allow one to correctly represent metastable systems, in which, by definition, phase equilibria are not steady-state. We believe that extended energy diagrams (EED) showing relationships between the crystalline phases constituting a system, incl. eutectics, can provide the most visual way to demonstrate the totality of thermochemical properties of systems.

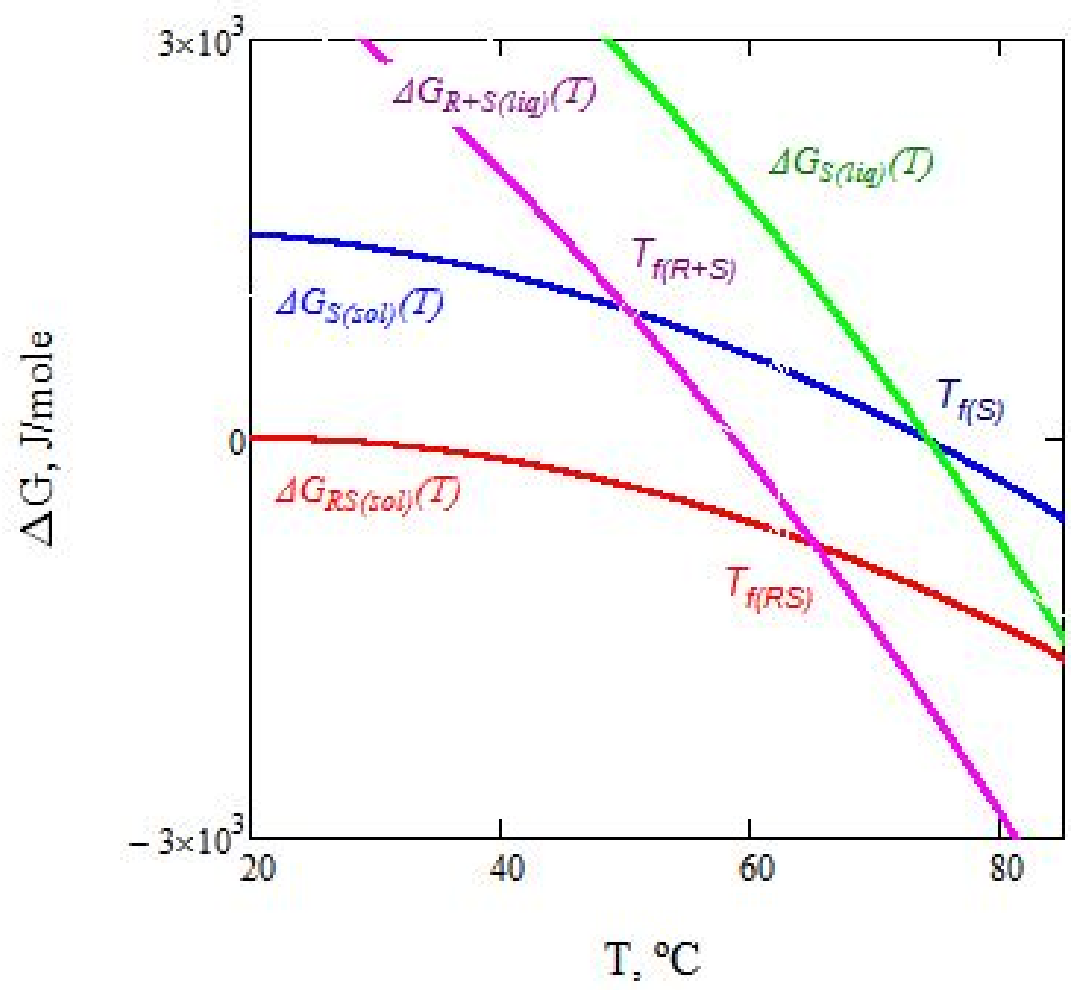

Figure S4. Typical dependence of the change in the free energies for binary mixture of stereoisomers on temperature: red curve - racemic compound, blue curve - enantiopure phase, green curve and magenta curve - racemic and enantiopure melts, respectively. The curves are extrapolated to entire temperature range.

Figure S4 shows a conventional energy diagram in its traditional form showing the relationship between the free energies of some crystalline enantiopure substance R (blue curve), crystalline racemic compound RS (red curve), enantiopure melt R (green curve), and racemic melt RS (purple curve).

In this case, the Gibbs energy values are measured relative to a hypothetical system for which the enthalpy and entropy are equal to the enthalpy and entropy of the crystalline phase RS under standard conditions. Such families of curves can be constructed based on the thermochemical 
parameters of individual phases obtained by the DSC method and the dependences of their heat capacity on temperature. In Figure S4, as in similar Figures S1-S3, the intersection of a pair of curves representing the temperature dependence of the free energies of the enantiopure crystalline phase (blue curve) and enantiopure melt (green curve) corresponds to the melting temperature of the enantiopure substance $T_{f(R)}$, and the curves for racemic compound (red curve) and racemic melt (purple curve) - the melting point of the racemic compound $T_{f(R S)}$. The intersection of the curves of the enantiopure substance and the racemic melt corresponds to the melting point of the conglomerate, that is, the melting point of the eutectic of stereoisomers. Obviously, in this conditional system, the latter can be observed only under conditions of metastability, since the racemic compound is thermodynamically preferable and melts at a higher temperature. But in a similar way, information can be extracted from the diagram for other characteristic compositions of a given system, such, for example, as the eutectic of the racemic compound and the enantiopure substance.

For an enantiopure substance $\mathrm{R}$ in contact with a scalemic melt $\mathrm{R}+\mathrm{S}$ with a molar fraction of this enantiomer $x_{R}$, the melting point (liquidus temperature) can be found from the condition that the free energies of the crystalline phase and the melt are equal. The free energy of the $R$ component in a mixed melt of composition $x_{R}$ is calculated as

$$
\Delta G_{x R(l i q)}\left(x_{R}, T\right)=\Delta G_{R(l i q)}(T)+R T \ln x_{R}
$$

where $\Delta G_{R, x_{R}=1}(T)$ is the temperature dependence of the free energy of the enantiopure melt, reflected by the green curve in Figure S4.

Varying the $x_{R}$ values generates a family of curves whose intersections with the curve for the crystalline enantiopure phase give the corresponding temperatures, which can be considered as liquidus points for the $\mathrm{R}$ phase in the phase diagram of the binary $\mathrm{R}+\mathrm{S}$ system (Figure $\mathrm{S} 5$ ). 

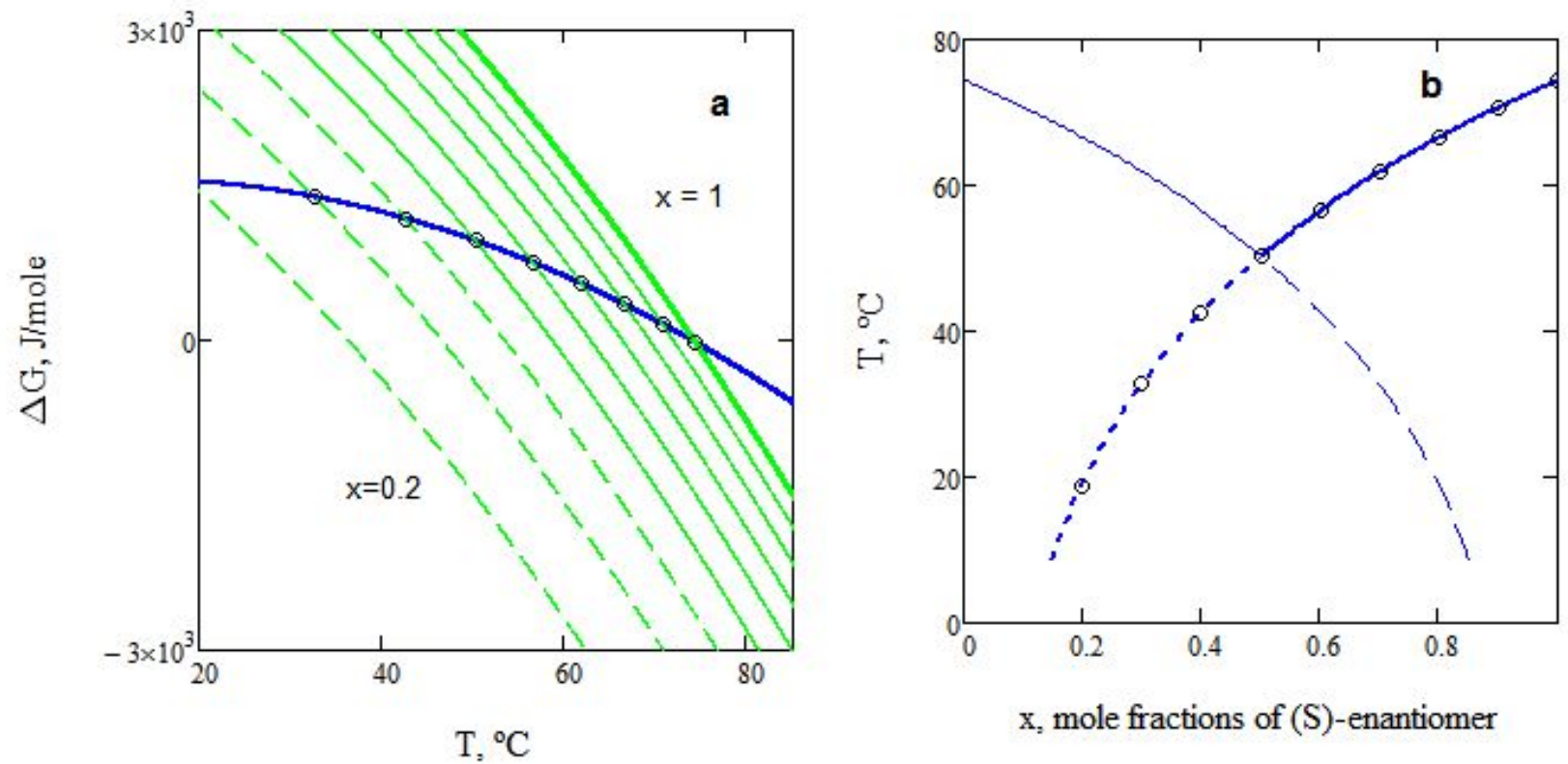

Figure S5. a. Ratios between the free energies of an enantiopure substance (blue curve) and the melt of this substance with different enantiomeric composition (family of green curves. The upper (bold) curve corresponds to an enantiopure melt, the dotted line marks the lines corresponding to experimentally unobserved equilibria between the crystalline sample and the melt at $x<0.5$. b. The same data presented in the coordinates temperature vs mole fraction form a binary phase diagram of the melting of the system under consideration.

Further, on the basis of the presented energy diagram Figure S4 data, the liquidus line of the phase diagram of a (hypothetical) conglomerate can be constructed as a numerical solution of equation 2 with respect to $\mathrm{T}$ for a given value of $x_{R}$ :

$$
\Delta G_{R(s o l)}(T)=\Delta G_{R(l i q)}(T)+R T \ln x_{R}
$$

Similarly, the thermodynamic potential of a mixture of enantiomers in a 1:1 ratio for a melt of composition $x_{R}$ is described by the equation:

$$
\Delta G_{x R S(l i q)}\left(x_{R}, T\right)=\Delta G_{R(l i q)}(T)+R T \ln \left[x_{R}^{1 / 2}\left(1-x_{R}\right)^{1 / 2}\right]
$$

This in turn allows the construction of a family of curves whose intersections with the free energy versus temperature curve for the crystalline phase of the racemic compound (red curve in Figure S4) correspond to the liquidus points of that phase in the phase diagram (Figure S6a). 

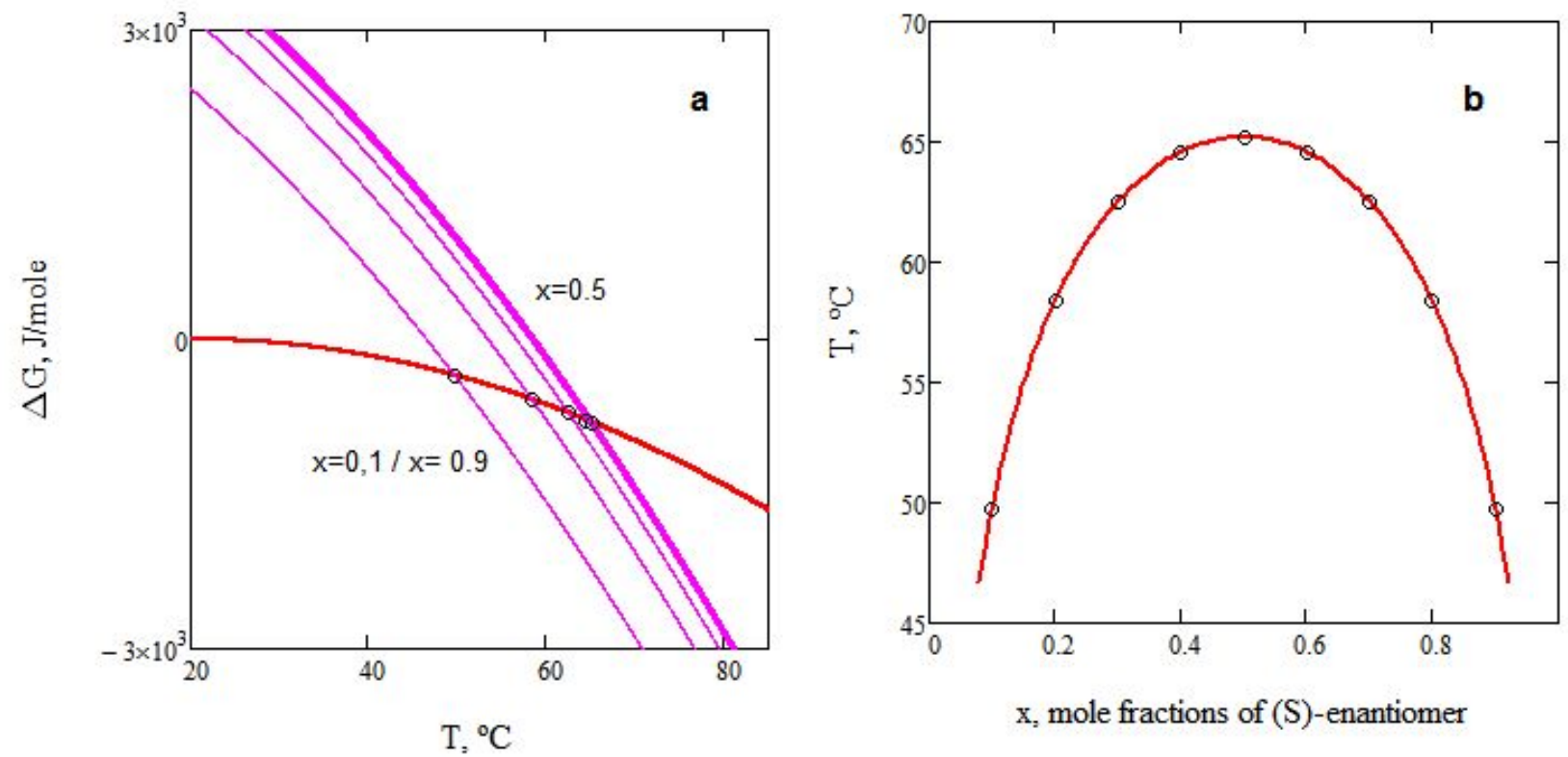

Figure S6. a. Temperature dependence of the free energy of a crystalline racemic compound (red curve) and a mixture of enantiomers in a binary melt of different enantiomeric composition (family of purple curves). The upper (bold) curve corresponds to the melt of an individual racemic compound. b. The liquidus branch of the binary phase melting diagram constructed on the basis of the same data.

Based on the same arguments, the liquidus line of the RS phase can be constructed as a numerical solution with respect to $\mathrm{T}$ for a given $x_{R}$ of equation (4):

$$
\Delta G_{R S(s o l)}(T)=\Delta G_{R(l i q)}(T)+R T \ln \left[x_{R}^{1 / 2}\left(1-x_{R}\right)^{1 / 2}\right]
$$

A joint solution of the combined equations (2) and (4) yields a pair of values (x, T) for which the curves of the dependence of the free energy of crystalline forms R and RS on temperature intersect with the curves of the thermodynamic potentials of the melt corresponding to the value of $x$ at temperature $\mathrm{T}$, which in turn corresponds to the simultaneous congruent melting of a mixture of crystalline phases R and RS in contact with a melt of a given composition at a constant temperature, or to the eutectic point of a given system (Figure S7). 

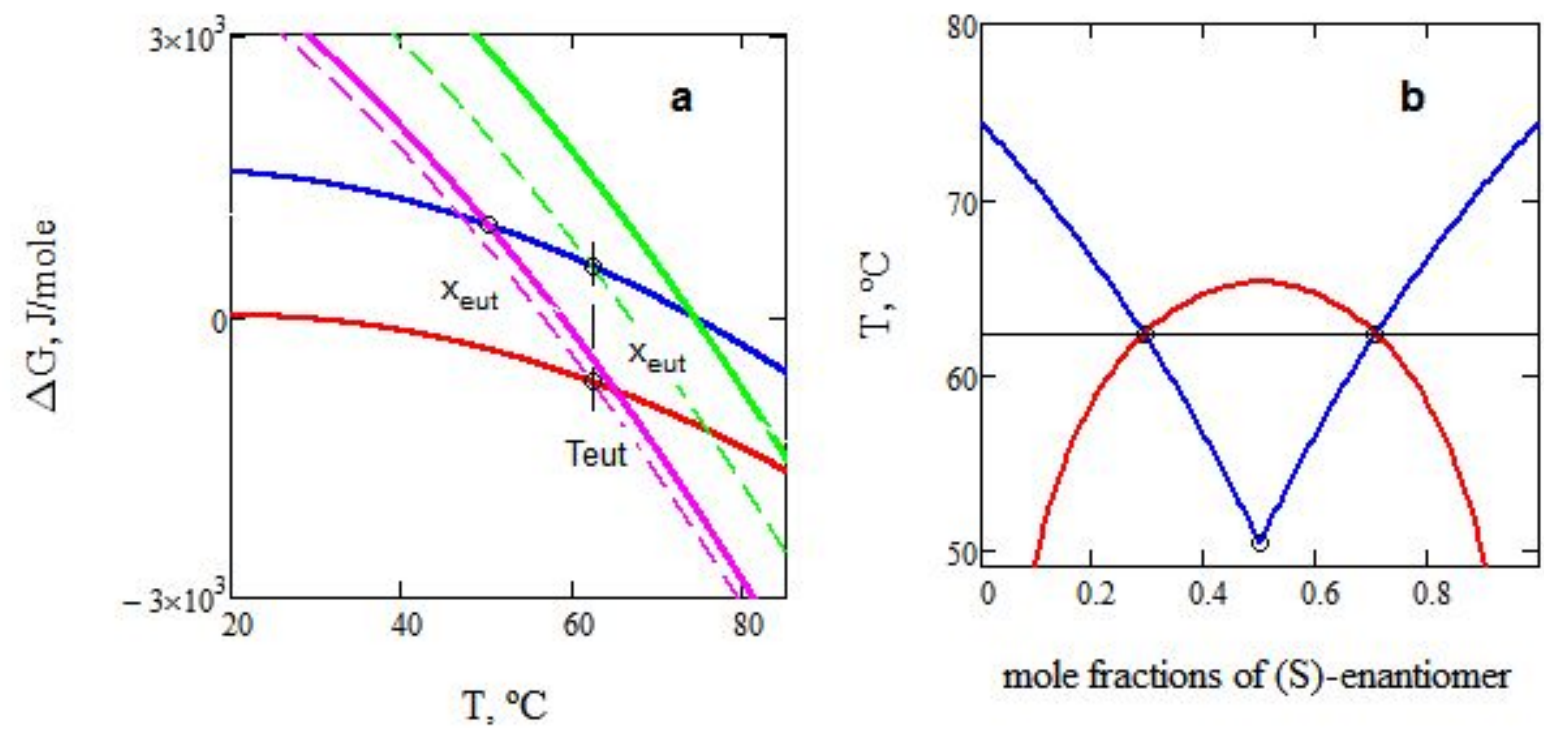

Figure S7. a. Extended energy diagram of the binary system presented in Figure S4. Dotted green and purple lines are, respectively, the thermodynamic potential of an individual enantiomer and a mixture of enantiomers in a melt of composition corresponding to the eutectic of their crystalline phases. b. The same data, presented in the coordinates "temperature vs mole fraction" form; a typical phase diagram of a binary mixture of stereoisomers.

All of the above retains its value in the transition to real tasks. Thus, solving the corresponding equations for stable and metastable phases of chlorphenesin $\mathbf{1}$ using real temperatures and free energies presented in Figure S1 allows us to construct an expanded energy diagram presented in Figure S8. 


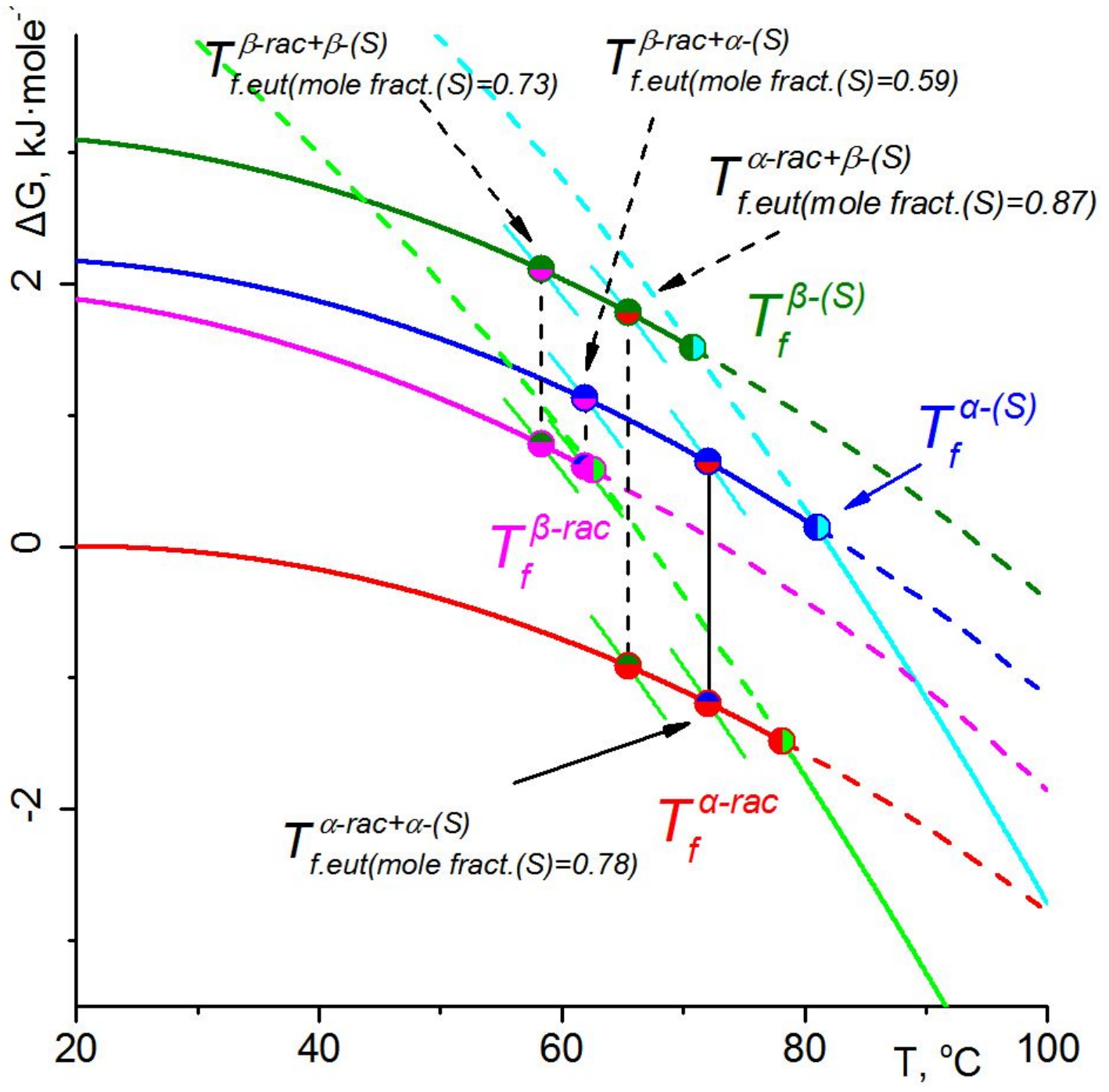

Figure S8. Dependence of the change in the free energies of the phases identified in system 1 on temperature: red curve - $\alpha-r a c-1$, magenta curve - $\beta$-rac-1, blue curve $-\alpha-(S)-\mathbf{1}$, olive curve - $\beta-(S)$ 1, green curve and cyan curve - racemic and enantiopure melts, respectively. In the region indicated by the dotted line, each of the phases is thermodynamically unstable; phases $\beta$-rac-1 and $\beta-(S)-\mathbf{1}$ are metastable throughout the interval where they are observed.

In this and all subsequent figures, the points connected by black vertical lines correspond to possible eutectics in the system under consideration between racemic and enantiopure phases. Vertical dashed lines denote metastable eutectics, vertical solid line - eutectics of thermodynamically stable phases. The pairs of cyan and green segments connected by vertical lines represent fragments of the curves of thermodynamic potentials, respectively, of an individual enantiomer and a 1:1 mixture of enantiomers for eutectic melts. 
An expanded energy diagram for the stable and metastable phases of diol 2, plotted from the temperatures and free energies shown in Figure S2, is shown in Figure S9.

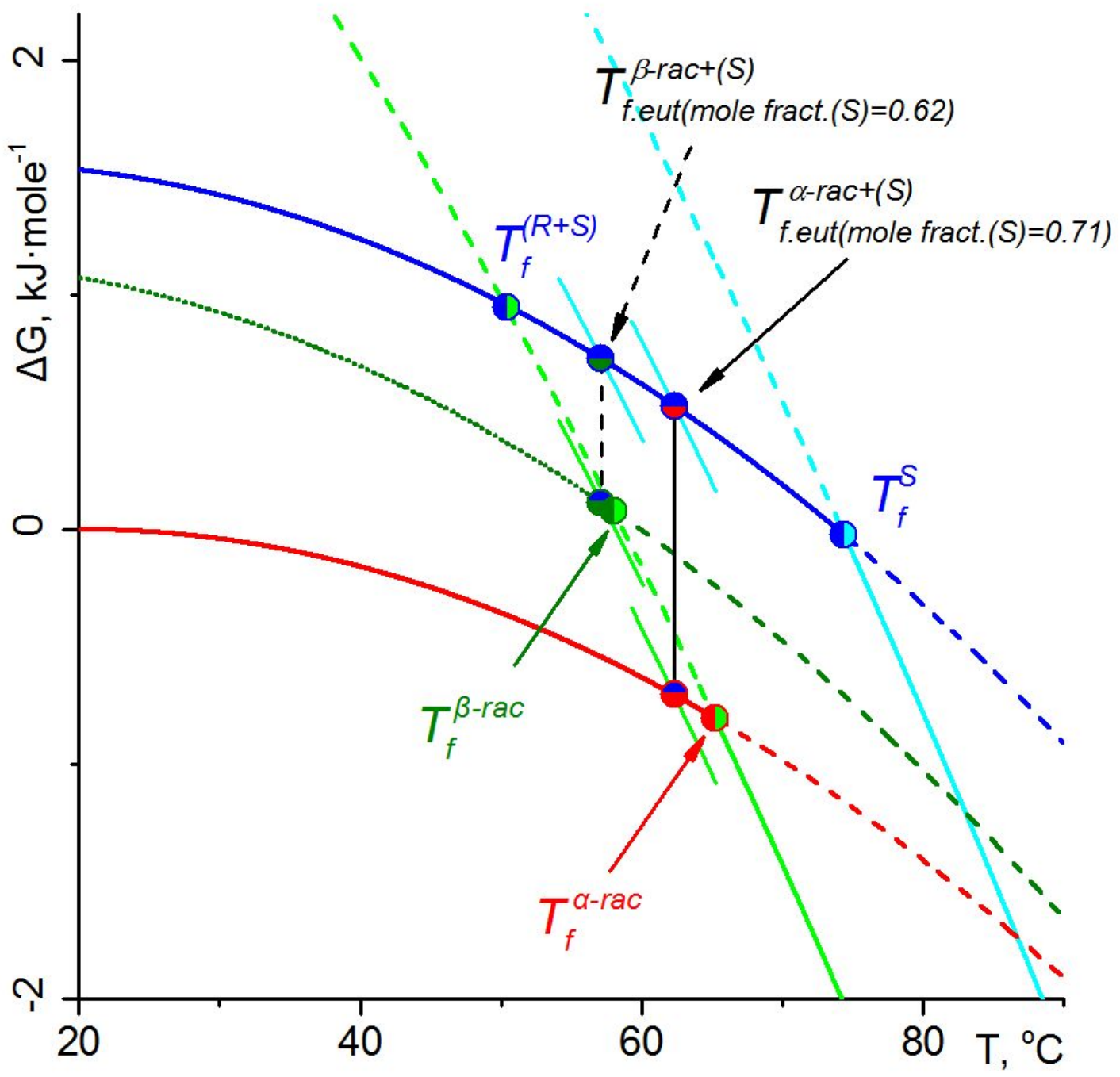

Figure S9. Dependence of the ratio of free energies of phase states of compound $\mathbf{2}$ on temperature: $\alpha-r a c-2$ (red curve), $\beta$-rac-2 (olive curve), enantiopure $S$-isomer (blue curve), racemic melt (light green curve), scalemic melt (light blue curve). In the region indicated by the dotted line, each of the phases is thermodynamically unstable; the $\beta-r a c-2$ phase is metastable throughout the interval where it is observed. Other designations are similar to those shown in Figure S8. 
An expanded energy diagram for stable and metastable phases of diol 3, plotted from the temperatures and free energies presented in Figure S3, is shown in Figure S10.

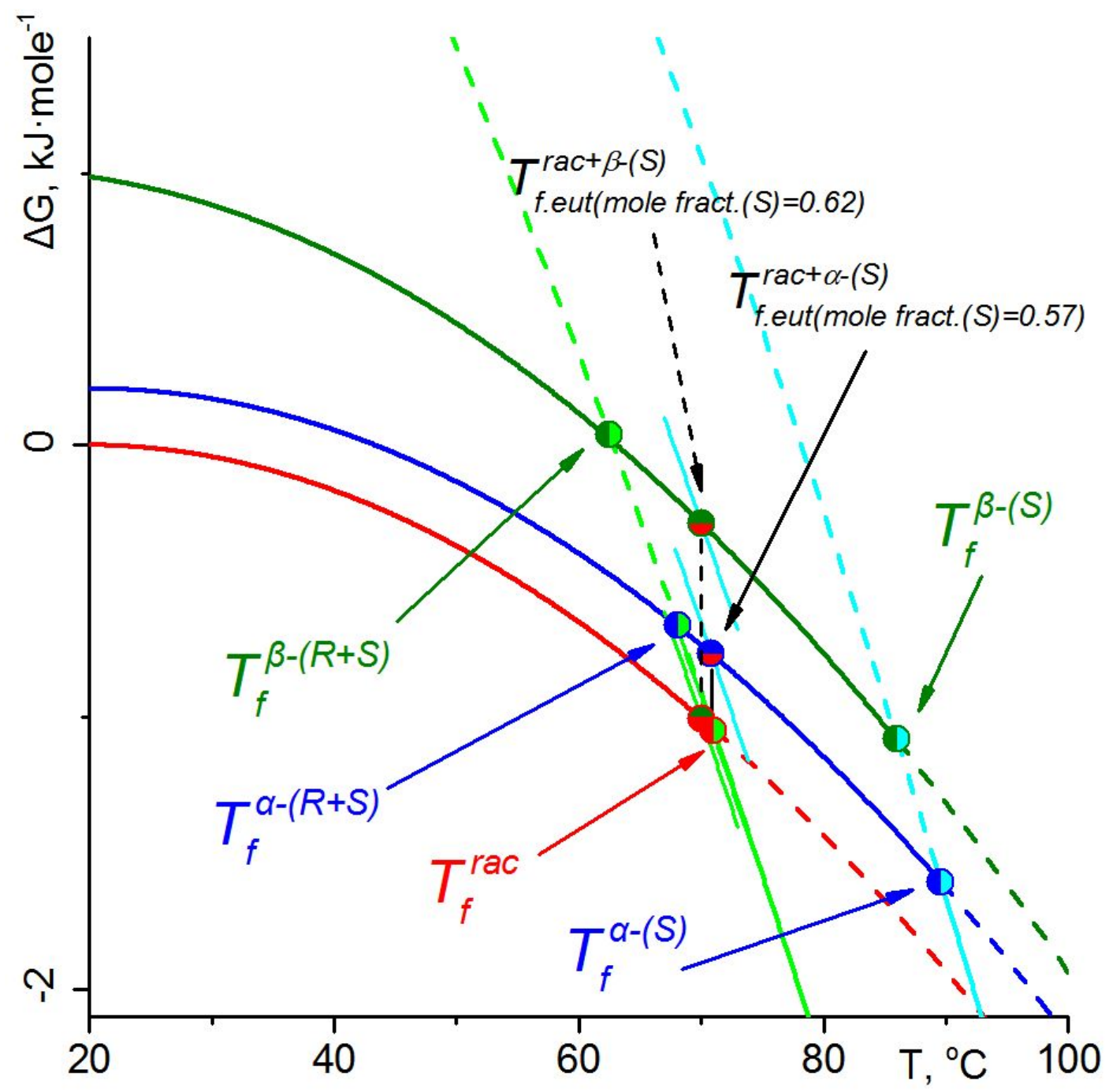

Figure S10. Dependence of the free energy ratios of diol $\mathbf{3}$ phase states on temperature: $\alpha-r a c-3$ (red curve), $\alpha-(S)-3$ (blue curve), $\beta-(S)-3$ (olive curve), racemic melt (green curve), scalemic melt (light blue curve). In the region indicated by the dotted line, each phase is thermodynamically unstable. The $\beta-(S)-3$ phase is metastable throughout the interval where it is observed. Other designations are similar to those used on Figures S8-S9.

We believe that energy diagrams of this type (EED) give a visual representation of the position of all possible points of eutectic melting on the temperature scale, and, accordingly, of the thermal effects that can be observed on DSC thermograms of samples of the considered system of any composition, incl. for nonequilibrium, with the participation of metastable phases. 
In addition to what has been said, it should be clarified that the height of the vertical segment connecting each of the pairs of points, representing the eutectics, can be considered as an objective quantitative characteristic of the energy gain in the formation of a given racemic compound from a mixture of enantiomers represented by a given enantiopure crystalline modification. This method of quantifying the energetics of formation of a racemic compound based on thermochemical data is similar to that proposed in the well-known work of Grant et al., ${ }^{4}$ but is more consistent, since it all follows the use of a single procedure for selecting characteristic points (eutectics) on the temperature scale for any systems and does not require changing the calculation method for this objective characteristic depending on the ratio of the melting points of the enantiopure phase and the racemic compound.

\section{References}

1. Bredikhin, A. A.; Zakharychev, D. V.; Bredikhina, Z. A.; Kurenkov, A. V.; Krivolapov, D. B.; Gubaidullin, A. T. Spontaneous resolution of chiral 3-(2,3-dimethylphenoxy)propane1,2-diol under the circumstances of an unusual diversity of racemic crystalline modifications. Cryst. Growth Des. 2017, 17, 4196-4206. DOI: 10.1021/acs.cgd.7b00510

2. Bredikhin, A. A.; Zakharychev, D. V.; Gubaidullin, A. T.; Bredikhina, Z. A. Solid phase behavior, polymorphism, and crystal structure features of chiral drug Metaxalone. Cryst. Growth Des. 2018, 18, 6627-6639. DOI: 10.1021/acs.cgd.8b00874

3. Bredikhin, A. A.; Zakharychev, D. V.; Bredikhina, Z. A.; Kurenkov, A. V.; Samigullina, A. I.; Gubaidullin, A. T. Stereoselective crystallization of chiral 3,4-dimethylphenyl glycerol ether complicated by plurality of crystalline modifications. Crystals 2020, 10, 201. DOI: $10.3390 /$ cryst 10030201

4. Li, Z. J.; Zell, M. T.; Munson, E. J.; Grant, D. J. W., Characterization of racemic species of chiral drugs using thermal analysis, thermodynamic calculation, and structural studies. $J$. Pharm. Sci., 1999. 88, 337-346. DOI: 10.1021/js980205u 